\title{
Observing Membrane Proteins via SANS During Lipidic Cubic Phase Crystallization
}

Thomas E. Cleveland IV, Paul Butler

NIST Center for Neutron Research

Membrane proteins can be incorporated into the lipidic cubic phase (LCP) for crystal growth and structure determination. LCP crystallization has become an important tool in the field of membrane protein crystallography (particularly, but not solely, with GPCRs). However, many details of this process are not well understood. There is little direct experimental evidence for the localization of protein and detergent after incorporation into LCP; the mechanisms of nucleation and crystal growth; and the details of how the cubic phase modifies the interactions between protein molecules. We are using Small Angle Neutron and X-Ray Scattering (SANS/SAXS) to study the LCP crystallization process, using Bacteriorhodopsin (bR) as a model system.

Using SANS, it is possible to contrast-match the non-protein components of the system, i.e. detergents and lipids. This allowed us to measure the scattering from bR directly and in isolation even after incorporation into the LCP, greatly simplifying the data interpretation from these complex multicomponent systems. At high bR concentrations in solution, it is also possible to measure structure factors, from which information on protein-protein interactions can be obtained. We have measured the concentration-dependent scattering of bR: (1) in solution; (2) after incorporation into LCP; and (3) as a function of precipitant concentration. Solution structure factor measurements at low salt concentrations were consistent with a repulsive charged sphere interaction model. In contrast-matched LCP at low concentrations of $b R$ and precipitant, scattering from $b R$ monomers was observed, similarly to $b R$ in solution. We also directly observed the dissociation of detergent micelles upon incorporation into the LCP. At higher bR and precipitant concentrations (but still below levels where crystals grew), larger bRcontaining structures were observable by SANS. At the highest precipitant concentrations, proteindependent Bragg peaks appeared in SANS measurements from samples in which macroscopic bR crystals later grew. 